\title{
Performance of deterministic local sensing aggregation under interference
}

\author{
Amin Nahvi \\ School of Electrical Engineering \\ KTH, Royal Institute of Technology \\ Stockholm, Sweden \\ Email: nahvi@kth.se
}

\author{
Viktoria Fodor \\ Access Linnaeus Center \\ KTH, Royal Institute of Technology \\ Stockholm, Sweden \\ Email: vfodor@ee.kth.se
}

\author{
Ioannis Glaropoulos \\ Access Linnaeus Center \\ KTH, Royal Institute of Technology \\ Stockholm, Sweden \\ Email: ioannisg@ee.kth.se
}

\begin{abstract}
In this paper we evaluate the delay and sensing performance of distributed cooperative sensing in wireless sensor networks assisting cognitive secondary operation. We propose a time division multiple access protocol to exchange sensing information among neighboring sensors, that is tunable in terms of sensing cooperation range and frequency reuse distance. Our evaluation shows that short frequency reuse distance may cause significant packet loss in the sensing aggregation process. This loss however affects mainly transmissions with less significant information, and therefore low delay aggregation with efficient spatial time division multiplexing is possible.
\end{abstract}

\section{INTRODUCTION}

Cognitive radio technology provides the opportunity for unlicensed users to adapt their transmission parameters according to the environment and utilize in this way the unused portions of the licensed spectrum. However, the cognitive users themselves can not reliably detect the spectrum holes due to attenuation impairments, and this unreliability threatens the primary network performance [1]. The reliable detection of spectrum holes requires cooperative sensing, that is, spectrum sensing at several locations and the combination of the sensed information of the nearby sensors [2].

To ensure reliable cooperative sensing [3] proposes the use of a dedicated wireless sensor network (WSN) to assist the detection of spatiotemporal free spectrum bands. Figure 1 demonstrates this general framework. The sensor network monitors the spectrum, generates a map of occupied frequencies by performing cooperative sensing, and delivers it to the cognitive secondary network. The secondary network then tunes its transmission parameters accordingly. The sensing and communication performance of the WSN affect the interference caused in the primary network and the utilization of the available spectrum in the secondary cognitive network. The interference depends on the probability that a primary signal is not detected, called missed detection, and the reaction time of the WSN, including the sensing time and the time for sensing information fusion. The utilization of the available spectrum in turn depends on the probability of a false alarm, when a free frequency is detected as occupied, and again on the reaction

This work was supported in part by the European Community's Seventh Framework Programme (FP7/2007-2013) under grant agreement no. 216076 FP7 (SENDORA) and by the CROPS-II Nordite Programme.

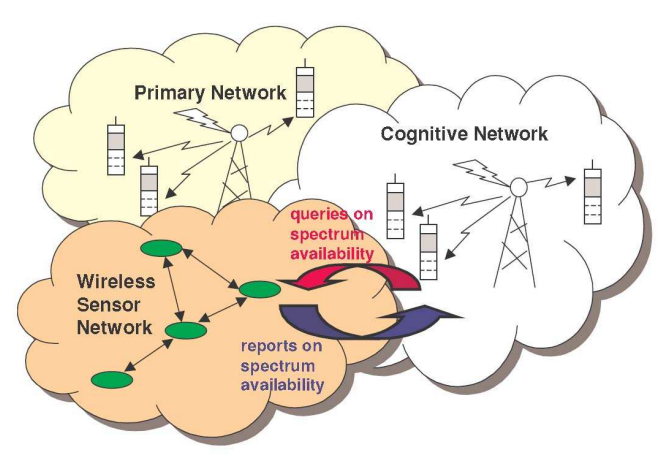

Fig. 1. WSN aided cognitive operation. A regular sensor grid is implemented to provide spectrum availability information for the secondary cognitive network [3]

time of the WSN, the faster is the sensing and information fusion, the longer is the time that remains to actually utilize the detected spectrum holes.

The sensing and communication performance of the WSN depends on several factors, such as the spectrum sensing mechanism applied and the cooperative sensing rule [4]; the WSN density and topology [5], and the WSN transmission capacity and protocol stack.

In this paper we address the local information aggregation process among the sensors, that is, when neighboring sensors exchange their sensing information and perform cooperative decision. These decisions are then collected by the fusion center in centralized architectures, or directly by the secondary users under distributed approaches.

We consider the scenario investigated in [5], where sensors of a fixed, regular grid perform energy detection over a set of bands, and combine their binary, spectrum free or spectrum occupied hypotheses with their neighbors' results following the OR decision rule. The choice of hard decision combining is motivated by the expectation that the capacity available for the WSN communication will be low and therefore the WSN traffic load has to be minimized. We recall, that the results of [5] show the existence of an optimal cooperation range, which maximizes the performance in terms of false 
alarm and missed signal detection tradeoff. As the cooperation range is further increased, sensors far away from the sensed physical point contribute to false alarms, but do not improve the signal detection performance significantly, which leads to overall sensing performance degradation.

Since the aggregation of sensing results in the fixed grid and within a given cooperation range is a deterministic process, we propose time division multiple access (TDMA) with spatial frequency reuse for the medium access control of the local aggregation. We evaluate the tradeoff of the data aggregation delay and the probability of packet loss due to interference, as a function of the sensing cooperation range and the frequency reuse distance of TDMA scheme, and the effect of packet losses on the cooperative sensing performance.

The rest of the paper is structured as follows. Section II presents the networking scenario, that is, the cooperative sensing process and the TDMA based aggregation. Section III evaluates the performance of the proposed schemes and derives the optimal parameters of the cooperative sensing and the channel access protocol. Section IV concludes our work.

\section{SYSTEM DESCRIPTION}

We consider a wireless sensor network that is deployed in the area where cognitive operation is allowed, forming a regular triangular grid. The cognitive operation range is divided into multiple bands, and each sensor performs spectrum sensing over the set of bands to find the temporarily free ones. The WSN and the secondary network operations are synchronized, and follow a fixed schedule. At the beginning of each time-slot the WSN performs spectrum sensing and provides spectrum availability estimates for the secondary network. After this period, the secondary network performs cognitive transmission on the temporarily free bands until the end of the time-slot. The length of the time-slot determines the maximum time of interference due to missed detection, and is set according to the requirements of the primary technology.

\section{A. Cooperative sensing with local aggregation}

Each sensor is responsible for detecting active primary transmitters in its Voronoi cell. In the case of the considered triangular grid, the Voronoi cell is a hexagon as shown in Figure 2. An active primary transmitter in a Voronoi cell is not detected if the respective sensor decides for signal not present after the cooperative sensing process. Similarly, false alarm occurs, if the sensor decides for signal present while there is no transmitter in the cell.

The local sensing process is controlled by the detection threshold $\gamma$, and is set to limit the false alarm probability after cooperative decision, based on the preliminary knowledge of the noise variance, $\sigma^{2}$, the number of samples, $K$, and the number of cooperating sensors, $N_{c}$. Considering OR decision, the model of [5] is used to calculate $\gamma$ :

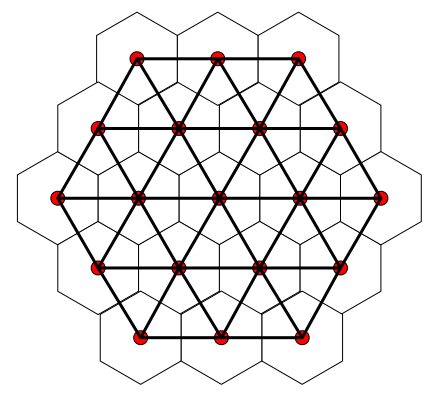

Fig. 2. The triangular grid of sensors with hexagonal Voronoi cells. The first sensor tier consists of 6 , the second sensor tier consists of 12 sensors.

$$
\begin{aligned}
p_{F A} & =1-\left(1-p_{f a}\right)^{N_{c}}, \\
p_{f a} & =Q\left(\frac{\gamma-\sigma^{2}}{\sqrt{\frac{2 \sigma^{2}}{K}}}\right) .
\end{aligned}
$$

The sensors exchange their set of local decisions with the neighboring nodes up to the cooperation range, $R_{c}$, defined as the number of sensor tiers, in WSN hops. For instance, if $R_{c}=1$, sensors exchange information with the first tier, that is, the six one hop neighbors. If $R_{c}=2$, information is exchanged with the first and second tier, including one and two hop neighbors respectively. Figure 2 shows the triangular grid. The first tier consists of 6 , the second of 12 sensors. That is, cooperative decision with $R_{c}=2$ combines $1+6+12=19$ measurements. In general, the number of cooperating sensors for given $R_{c}$ and for triangular grid is equal to:

$$
N_{c}=3 R_{c}^{2}+3 R_{c}+1 \text {. }
$$

The vector of local binary decisions, representing the availability of the set of sensed bands, is protected with error detection code. If a node can not decode a transmission due to interference, the packet is considered to be lost. Lost local decisions are considered as signal not present in the OR decision. Consequently, packet loss increases the probability of missed detection and decreases the probability of false alarm. That is, the limit on the false alarm probability $p_{F A}$ holds even under lossy transmission.

\section{B. Medium access control for local aggregation}

We assume that sensors share their local decisions with direct broadcasting. That is, when a sensor broadcasts its local binary decision, all other sensors within $R_{c}$ listen to receive the information.

Since the information exchange between the sensors of the fixed grid leads to a deterministic network load, we base the channel access on spatial time division multiple access (TDMA). Each time-slot of the TDMA frame is assigned to a group of sensors. Sensors broadcast the vector of their local 


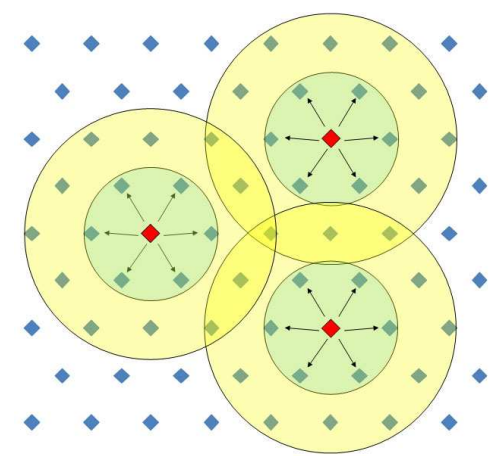

Fig. 3. The TDMA schedule. $R_{c}=1$ and $R_{i}=2$.

binary decisions over the sensed channels in the assigned timeslot, and all sensors within the cooperation range make an attempt to receive the information. By the end of a TDMA frame each sensor has broadcasted its local estimate and can make the cooperative decision based on the received information.

The delay of this local aggregation process is determined by the length of the TDMA frame, that is, by the required number of time-slots. Consequently, the TDMA slot assignment should minimize the number of timeslots provided the requirements on the sensing performance are fulfilled.

Since the transmission frequency is reused, packet losses may happen due to the interference caused by simultaneous transmissions. To control the level of interference, we introduce the tunable system parameter interference range, $R_{i}$, which gives the minimum distance between a receiving and a simultaneously transmitting node in hops. Clearly, $R_{i} \geq R_{c}$ is needed to keep the interference power below the signal power at the receiving node. Figure 3 demonstrates the concepts of interference and cooperation range for $R_{c}=1$ and $R_{i}=2$. The inner and outer discs represent the cooperation and interference ranges respectively. It is reasonable that the interference areas overlap, since nodes in the overlapping areas are not included in the cooperation areas and are not expected to receive the packets. To keep the interference range limit, the TDMA slot assignment has to be such that $k$, the distance of nodes transmitting at the same time, is:

$$
k \geq R_{i}+R_{c} .
$$

Clearly, larger interference range decreases the interference and consequently the packet loss probability, while increases the time required for the information aggregation.

For an arbitrary sensor network structure, the construction of the minimum length TDMA schedule that obeys (3) is equivalent to the Distance- $k$ Chromatic Number Problem.

The Distance- $k$ Chromatic Number Problem is defined as follows. Given a graph $G=(V, E)$, and an integer $k$, the distance- $k$ chromatic number of the graph is the fewest number of colors needed to color the nodes of the graph so that no two nodes of the graph have the same color if the shortest path length between the nodes is less than or equal to $k$. Such a
TABLE I

Simulation PARAMETER VALUES

\begin{tabular}{|c|c|}
\hline \multicolumn{2}{|l|}{ Sensing } \\
\hline False alarm probability $p_{F A}$ & 0.05 \\
\hline Sensed bandwidth & $200 \mathrm{KHz}$ \\
\hline Sensing time & $1 \mathrm{~ms}$ \\
\hline \multicolumn{2}{|c|}{ Wireless Sensor Network } \\
\hline Nodes distance $d$ & $50 \mathrm{~m}$ \\
\hline Cooperation range $R_{c}$ & $1-3$ \\
\hline Interference range $R_{i}$ & $2-6$ \\
\hline Packet size & 4Bytes \\
\hline Modulation & BPSK \\
\hline Transmission frequency & $2.5 \mathrm{GHz}$ \\
\hline Transmission bandwidth & $10 \mathrm{KHz}$ \\
\hline Transmission power & $17 \mathrm{dBm}$ \\
\hline \multicolumn{2}{|l|}{$\begin{array}{l}\text { Primary network } \\
\end{array}$} \\
\hline Transmission frequency & $2.437 \mathrm{GHz}$ \\
\hline Transmission bandwidth & $20 \mathrm{MHz}$ \\
\hline Transmission power & $17 \mathrm{dBm}$ \\
\hline \multicolumn{2}{|c|}{ Channel characteristics } \\
\hline Shadowing standard deviation & $10 \mathrm{~dB}$ \\
\hline Noise power density $\sigma^{2}$ & $-174 \mathrm{dBm}$ \\
\hline
\end{tabular}

coloring of the nodes of the graph is known as proper coloring. In the considered TDMA scheme the colors can be regarded as slot numbers and proper coloring gives a minimum length TDMA schedule.

The Distance- $k$ Chromatic Number Problem is NP-complete for the general case. For the triangular graph structure considered in this paper, we apply the sub-optimal solution in [6], originally proposed to solve the channel assignment problem in cellular networks. Here we customize the algorithm to find the minimum number of time slots for each $R_{i}$ and $R_{c}$ pair. The algorithm in [6] gives a slot assignment with

$$
n=k^{2}+k+1=\left(R_{c}+R_{i}\right)^{2}+R_{c}+R_{i}+1
$$

time-slots, so, the delay of the local aggregation increases quadratically with the sum of $R_{c}$ and $R_{i}$.

The time inefficiency of the aggregation is reflected by the difference of $n$ in (4) and $N_{c}$ in (2), since $N_{c}$, the number of cooperating nodes, gives the lower bound of the number of timeslots required to collect all information for the cooperative decision. The time inefficiency is due to the required interference range and due to the possible suboptimality of the slot assignment algorithm. Considering the $R_{i}=R_{c}$ limit, we can conclude that both the $n$ and $N_{c}$ values and therefore even their difference increase quadratically with $R_{c}$.

\section{PERFORMANCE EVALUATION}

In this section we evaluate the performance of the local aggregation process. First we consider network performance parameters like packet loss and aggregation delay, then even the performance of cooperative sensing, and evaluate how packet loss due to interference affects the signal missed detection probability.

We implemented the cooperative sensing algorithm and the spatial reuse TDMA MAC protocol in a simulator framework build on the ns2-miracle library [7]. To model the primary 


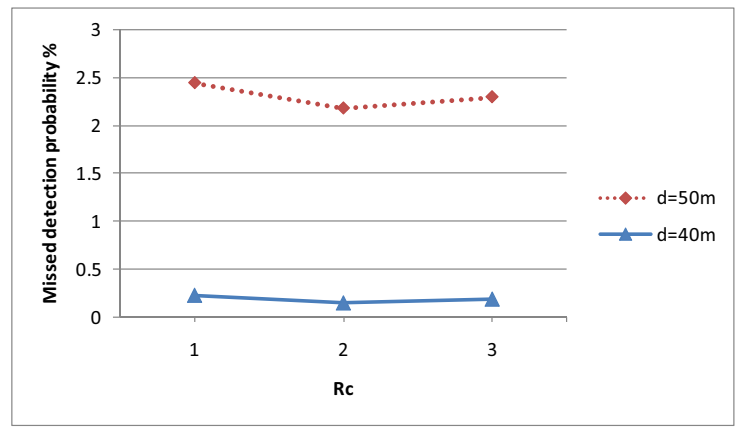

Fig. 4. Probability of missed detection without packet losses, for different sensor distances $d$ and as a function of the cooperation range $R_{c}$.

system we consider the transmission parameters of IEEE 802.11 WLAN [8]. The primary system and WSN parameters used for the simulation study are listed in Table I. Note that the system consists of two channels, one for primary transmissions and one for intra-WSN communication. For both channels we consider Gaussian noise, log-normal shadowing and Rayleigh fading with the same parameters.

We aim at evaluating the worst case signal detection probability and therefore simulate the transmission of primary packets from a transmitter located at the corner of a Voronoi cell. To set the local decision threshold $\gamma$ according to (1) we consider $p_{F A} \leq 5 \%$. For the considered sensed channel bandwidth and sensing time parameter values the number of samples is $K=400$.

To determine the network density and cooperation range values of interest, we first evaluate the WSN sensing performance without considering the eventual packet losses. Figure 4 shows the missed detection probability as a function of the cooperation range $R_{c}$. We consider $d=40 \mathrm{~m}$ and $d=50 \mathrm{~m}$. For both cases the optimal cooperation range is $R_{c}=2$. The performance gap in terms of missed detection probability is one order of magnitude between the two cases. For the considered scenario of WLAN as primary system $d=50 \mathrm{~m}$ provides acceptable performance, so we use this parameter value for the rest of the analysis and consider $R_{c}=1-3$.

Let us now investigate the WSN data aggregation performance in terms of packet loss ratio and data aggregation delay.

Figure 5 shows the packet loss ratio as a function of the interference range $R_{i}$, for $R_{c}=1-3$ and $R_{i} \geq R_{c}$. As we can see, $R_{i}$ has significant effect on the packet loss, that is, the system is in the interference limited regime. When the cooperation and interference ranges are equal, the packet loss ratio decreases with $R_{c}$, since large $R_{c}$ also means large frequency reuse distance.

From these results it seems that the interference range has to be large to achieve reasonable loss performance. Large interference range, however, increases the aggregation delay significantly. From Section II-A we recall though that sensing

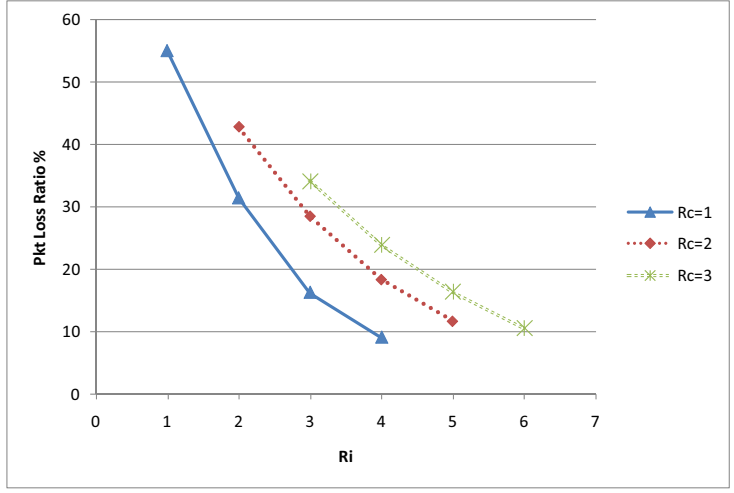

Fig. 5. Packet loss ratio as a function of the interference range for $R_{c}=1-3$, $d=50 \mathrm{~m}$.

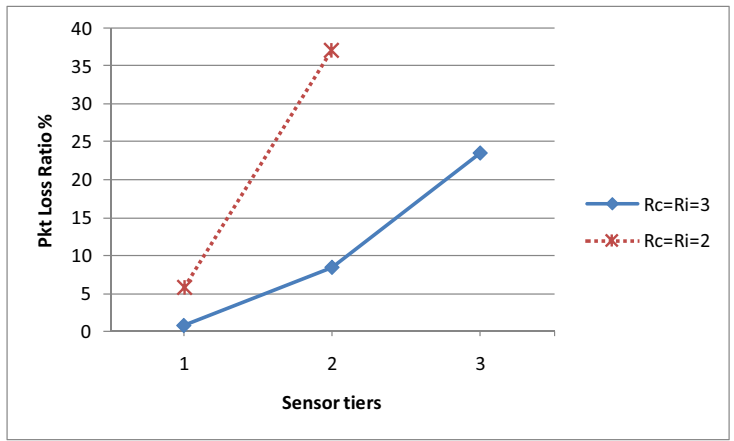

Fig. 6. Packet loss ratio from the different sensor tiers for $R_{c}=R_{i}=2,3$, $d=50 \mathrm{~m}$

results have different significance, depending on the sensor's distance from the sensed point. Specifically, second tier sensors contribute less to the cooperative decision than first tier sensors, and so on. Therefore, it is worth to investigate the distribution of losses among the sensor tiers. Figure 6 shows the packet loss ratio per sensor tier for $R_{c}=2,3$ and $R_{i}=R_{c}$. As we see, most of the losses occur in transmissions from sensors in the outer tiers, since the received signal level in this case is relatively low. This is a promising outcome for our scenario, since it might mean that losses do not cause significant sensing performance degradation and lower interference range values can be permitted.

Figure 7 shows the signal missed detection probabilities as a function of $R_{c}$ and $R_{i}$. If $R_{c}=1$ is used, losses have significant affect on the sensing performance, and therefore an interference range of $R_{i}=4$ has to be used to achieve a sensing performance similar to the no-loss case shown on Figure 4. Specifically, for $R_{i}=4$ the missed detection probability is $2.8 \%$, while by avoiding losses $2.21 \%$ can be achieved according to Figure 4. For higher cooperation ranges however, losses have very little effect due to two 


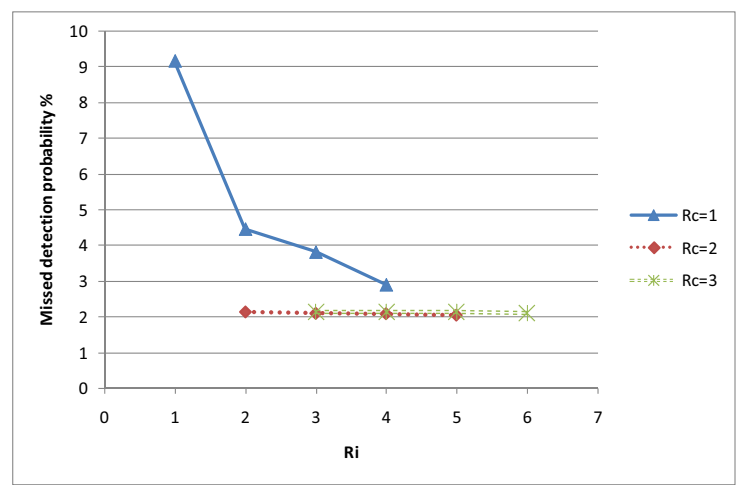

Fig. 7. Probability of missed detection under packet loss as a function of $R_{i}$ for $R_{c}=1-3, d=50 \mathrm{~m}$.

reasons. First, most of the losses happen in the less significant outer tiers. Second, as the second and third tiers have many sensors, receiving information from a part of them achieves the necessary cooperation gain. In our case for $R_{c}=2$ the achievable missed detection probability is $2.03 \%$, with $R_{i}=R_{c}=2$ it is $2.13 \%$, while with large interference range $R_{i}=5$ the missed detection probability is $2.04 \%$. Based on the results we can conclude that $R_{c}=R_{i}$ can be selected for $R_{c}>1$.

Finally, let us discuss the aggregation delay of the considered scenario. The aggregation delay is deterministic and is given by the length of the time-slot times $n$ in (4). The time-slot duration depends on the packet size, the modulation scheme and the transmission bandwidth and is calculated by the simulator. We set the packet size to 4Bytes, which includes the sensor identification number and the vector of binary decisions with an error detection code. For the considered modulation and WSN transmission bandwidth the time-slot duration on the inter-sensor communication channel is $6.4 \mathrm{~ms}$. If $R_{c}=1$ is selected, which might be a reasonable choice considering the little gain of $R_{c}=2$ on Figure $4, R_{i}=4$ provides sensing result close to the ideal, no-loss case, and therefore the required number of timeslots is $n=31$ according to (4). If, however, the optimal $R_{c}=2$ value is used, sensing quality close to the no-loss performance is achieved already at $R_{i}=R_{c}$, which leads to significantly decreased TDMA frame length with $n=21$. Based on the results, we conclude that $R_{c}$ and $R_{i}$ have to be optimized together to achieve the lowest delay under given sensing quality constraints.

\section{Discussion}

In this paper we presented a solution for local sensing information aggregation in low bandwidth WSN aiming at assisting cooperative sensing at the sensor nodes. In the proposed solution the information aggregation is based on spatial reuse TDMA, where the frequency reuse distance is selected based on the cooperation range of the cooperative sensing application and an interference range that limits the interference and consequently the packet loss due to simultaneous transmissions.

Based on simulations we evaluated the sensing and sensing aggregation performance of the WSN in terms of packet loss, aggregation delay and signal missed detection probability under false alarm probability constraint. Specifically, we considered primary system parameters corresponding to IEEE 802.11 WLAN systems. We concluded that the cooperation and interference ranges have to be jointly optimized to achieve fast aggregation with good sensing performance.

Since the presented results are based on specific system parameters, it is important to discuss how general the conclusions of the performance evaluation are. The WSN density, determined by the sensor distance $d$ depends heavily on the local sensing performance of the sensors, on the primary transmission parameters and on the primary transmission channel characteristics. Our previous paper [5] addresses this issue in detail.

The aggregation delay is determined by the cooperation and interference ranges. We believe that $R_{c}=2$ is the optimal cooperation range for many scenarios, since the second sensor tier consists of a high number of sensors which leads to sufficient sensing diversity. Even the results on the required interference range $R_{i}$ seem to be rather general, since for reasonable interference ranges the WSN works in the interference limited regime, where the exact values of the sensor transmission power and the receiver noise level do not significantly affect the data aggregation performance.

Therefore, we conclude that spatial reuse TDMA schemes provide an efficient solution for sensing information aggregation in regular WSNs, fulfilling the application requirements in terms of aggregation delay and cooperative sensing performance.

\section{REFERENCES}

[1] E. Larsson and M. Skoglund, "Cognitive radio in a frequency-planned environment: some basic limits," IEEE Transactions on Wireless Communications, , vol. 7, no. 12, December 2008.

[2] A. Ghasemi and E. Sousa, "Spectrum sensing in cognitive radio networks: requirements, challenges and design trade-offs," IEEE Communications Magazine, vol. 46, no. 4, April 2008.

[3] B. Mercier et al., "Sensor networks for cognitive radio: Theory and system design," in ICT Mobile Summit, June" 2008.

[4] T. Yucek and H. Arslan, "A survey of spectrum sensing algorithms for cognitive radio applications," IEEE Communications Surveys Tutorials, IEEE, vol. 11, no. 1, 2009.

[5] V. Fodor, I. Glaropoulos, and L. Pescosolido, "Detecting low-power primary signals via distributed sensing to support opportunistic spectrum access," in IEEE ICC, International Conference on Communications, June 2009.

[6] A. Sen, T. Roxborough, and S. Medidi, "Upper and lower bounds of a class of channel assignment problems in cellular networks," in IEEE INFOCOM, 1998.

[7] N. Baldo, F. Maguolo, M. Miozzo, M. Rossi, and M. Zorzi, "ns2-miracle: a modular framework for multi-technology and cross-layer support in network simulator 2," in ValueTools '07: International conference on performance evaluation methodologies and tools, 2007.

[8] "Sendora deliverable 2.1," EU FP7 SENDORA, www.sendora.eu, Tech. Rep., March 2008. 